\title{
Reflexiones a partir de un abordaje psicoantropológico para los estudios de población*
}

\author{
Carolina Martínez Salgado**
}

La investigación sociodemográfica de las últimas décadas ha puesto de manifiesta la necesidad de desarrollar abordajes transdisciplinarios para avanzar en la comprensión de la problemática de la que se ocupa. En este trabajo se discuten algunas de las posibilidades que se abren para los estudios de población, al incorporar ciertos elementos de tipo antropológico combinados con otros procedentes de la reflexión sociopsicoanalítica. Se trata de una propuesta para avanzar hacia la comprensión de las raíces individuales y familiares del comportamiento poblacional entendido como fenómeno específicamente humano, desde una perspectiva microsocial de naturaleza cualitativa. El problema que da lugar al ejercicio empírico se ubica en la intersección de tres cuestiones de gran relevancia para la problemática poblacional de nuestros días: la reproducción biológica, la relación con el entorno ambiental y los efectos de ambas circunstancias sobre la salud. La exploración abarca la unidad de análisis individual y la familiar, y en el proyecto se comparan una zona popular urbana y un pueblo tradicional en la zona de Xochimilco, en el sur de la ciudad de México.

En este trabajo planteo un conjunto de reflexiones surgidas durante el diseño de una investigación destinada a profundizar en la intersección de tres cuestiones de particular relevancia para la problemática poblacional de nuestros días, cuya discusión cristalizó en varias de las propuestas contenidas en diversos capítulos de la Agenda $21^{1}$ la reproducción biológica, la relación de la población con el entorno ambiental y la vinculación de estas dos esferas con los problemas de salud. El estudio se está desarrollando en un pequeño grupo de familias de nivel socioeconómico bajo, en dos áreas de Xochimilco, ${ }^{2}$ mediante un procedimiento que incorpora una combinación de elementos de la antropología y de los estudios psicosociales como complemento al enfoque sociodemográfico.

* El proyecto se lleva a cabo gracias a una beca del Fondo para la Formación de Líderes del Programa de Población de la Fundación MacArthur.

** Departamento de Atención a la Salud, UAM-Xochimilco.

1 Documento oficial de la Conferencia de Naciones Unidas sobre Medio Ambiente y Desarrollo, signada por los países asistentes a la "Cumbre de la Tierra", Río de Janeiro, junio de 1992.

2 Sobre el procedimiento para elegir las dos áreas de Xochimilco, puede revisarse Martínez, 1993. 
Este ejercicio es un intento de abordar algunos de los planos más profundos involucrados en el estudio del comportamiento poblacional, que surge de la preocupación por avanzar hacia un conocimiento más completo e integral de los procesos que este comportamiento implica. Pero no voy a referirme en estas páginas al problema de investigación o al ámbito en el que se lleva a cabo, sino a las posibilidades de complementariedad que encuentro en la confluencia de tres fecundas tradiciones -la sociodemográfica, la psicoantropológica y la sociopsicoanalítica- para afrontar el reto de penetrar en la comprensión de las raíces individuales y familiares del comportamiento poblacional.

La vertiente sociodemográfica: hacia los estudios en profundidad

En el año de 1986 apareció un volumen con los trabajos de un seminario organizado por el Programa de Investigaciones Sociales sobre Población en América Latina (PISPAL, 1986), en cuya introducción se relata cómo el seminario, inicialmente concebido para discutir los principales problemas del uso de técnicas demográficas en la investigación interdisciplinaria, terminó enfocándose a toda una gama de problemas teórico-metodológicos implicados en la integración del estudio de la dinámica demográfica dentro de la investigación social (Stern, 1986).

Las discusiones planteadas durante aquel seminario dejaron ver, entre muchas otras cosas, que las explicaciones del comportamiento demográfico logradas al analizar sus relaciones con las variables económicas y sociales más globales llegaban sólo hasta un cierto nivel, más allá del cual escapaban a los alcances de los estudios macro, e incluso de aquellos micro que se valían de técnicas como los censos y encuestas. De manera que una de las propuestas para los estudios de población sería la de integrar los aportes de diferentes tipos de aproximación y combinar los distintos niveles de análisis y estrategias metodológicas.

Del abanico de perspectivas presentes en aquel evento, hay algunas que constituyen importantes antecedentes para este estudio, por los campos problemáticos a los que apuntan. Entre ellas destacan la que reconsidera los alcances y limitaciones de las encuestas de actitudes y opiniones (Oliveira y García, 1986); la que se ocupa de las especificidades de la investigación microsocial (Jelin et al., 1986), y la que subraya, entre otras cosas, la importancia de la familia como instancia mediadora entre los planos sociales más globales y el comportamiento individual (Lerner y Quesnel, 1986). 
Las tres perspectivas reconocen, aunque con diferente énfasis, la importancia de los resultados obtenidos mediante técnicas como la encuesta por muestreo, de amplia utilización en la investigación sociodemográfica, pero las tres se manifiestan por la conveniencia de combinar distintos estilos de investigación ${ }^{3}$ y diferentes niveles de análisis (instituciones, familias, individuos) para entender cómo se gesta el comportamiento de las personas dentro del margen de opciones que sus circunstancias específicas les ofrecen. En los tres trabajos se subraya, siguiendo a Przeworski (1982), que las personas no necesariamente se comportan de manera homogénea sólo por pertenecer a grupos sociales similares. ${ }^{4}$ Los tres textos tocan, también, la cuestión de la dimensión temporal (tema que encontramos en varios de los documentos de ese volumen), aunque con matices que revelan las preocupaciones centrales de sus autores: Oliveira y García se refieren a las posibilidades de recuperarla a través de la atención a la edad, el ciclo de vida de los individuos y el ciclo vital familiar; Jelin et al. nos recuerdan que la temporalidad está involucrada no sólo en el problema que se investiga, sino también en la etapa por la que pasa la comunidad disciplinaria cuyos conocimientos se están poniendo en ịuego, y en los cambios que el avance del proceso de investigación va produciendo en el investigador mismo; Lerner y Quesnel centran su preocupación en las complejidades que supone el estudio de las relaciones entre la dinámica demográfica -y cada uno de sus componentes- con los demás procesos que forman parte del global de reproducción de la sociedad, cada uno de los cuales tiene sus propias temporalidades.

Además de estos planteamientos coincidentes, me interesa subrayar algunas cuestiones específicas que se recogen en cada uno de estos documentos. Una de ellas, la que se refiere a la familia como mediación entre los planos micro y macro (Lerner y Quesnel, 1986) será retomada un poco más adelante. Las otras

\footnotetext{
${ }^{3}$ Los de tipo cuantitativo, que captan las características económicas y sociodemográficas de los hogares y sus integrantes a través de técnicas como censos y encuestas, y los cualitativos, que examinan las relaciones entre individuo y sociedad, y profundizan en el significado que los sujetos dan a sus propias condiciones y comportamiento.

4 "Desde esta perspectiva (...) las familias y los individuos son vistos como agentes activos que en coyunturas específicas pueden organizarse para enfrentar y transformar sus condiciones materiales de existencia, y no se parte de una uniformidad de conducta ni un patrón familiar homogéneo al interior de un mismo grupo social. De esa manera las propias características sociodemográficas de las unidades domésticas y la dinámica intrafamiliar, pueden contribuir a fijar opciones diferenciales al interior de un sector social." (Oliveira y García, 1986: 71-72).
} 
dos, que nos conducirán a los planteamientos que desarrollo en la siguiente sección, son el cuestionamiento de Oliveira y García a los supuestos de las encuestas por muestreo de opiniones y actitudes, y los numerosos puntos de discusión que se abren a partir del texto de Jelin et al. sobre el procedimiento propio del estilo de investigación microsocial.

Las encuestas -señalan Oliveira y García- pretenden recoger elementos de la realidad a través de las verbalizaciones expresadas por los individuos en respuesta a preguntas en su mayoría cerradas, referidas a periodos previamente definidos, que se plantean durante una o dos visitas del entrevistador. Por lo que se espera de sus resultados parecería suponer que el entrevistado puede expresarse sobre un objeto externo sin ninguna vinculación ideológica o afectiva con aquél; que sus respuestas son indicadores de probabilidades de acción y no sólo declaraciones de intención y que todos los sectores sociales (o amplios grupos de población, al menos todos aquellos hacia los cuales va dirigida una encuesta por muestreo) comparten significados comunes en torno a los aspectos estudiados por la encuesta, no sólo entre ellos sino también con el investigador.

El estilo de investigación microsocial -dicen Jelin et al.- recupera la preocupación por descubrir la lógica o sentido de la acción de los sujetos como integrantes de un grupo social, con marcos de relaciones sociales y patrones culturales específicos que requieren descripciones densas y no superficiales (Geertz, 1992). Esta búsqueda de sentido no se limita sólo a las respuestas positivas o "veraces", sino que abarca a los silencios y ocultamientos, como si fueran una especie de "síntomas" o manifestaciones por interpretar. Las categorías analíticas con las que se inicia el estudio se siguen reelaborando durante todo el proceso de investigación, para favorecer el rastreo de nuevo material en forma cada vez más precisa y ajustada al sentido que los sujetos le otorgan en su mundo; los patrones o tipos de comportamiento encontrados se interpretan dentro de esa estructura de sentido. En este estilo de investigación, comentan los autores, se establecen relaciones personalizadas, empáticas, entre el investigador y el investigado, y la elaboración de una reconstrucción no prejuiciada del problema analizado requiere de una atención especial por parte del investigador.

Este conjunto de planteamientos de la reflexión sociodemográfica nos coloca a las puertas mismas de una trascendente problemática que había sido ya reconocida y tratada -aunque con referencia a otros aspectos del comportamiento humano- por la tradición que analizaré como segunda vertiente que alimenta mi propuesta. 
La vertiente psicoantropológica: hacia la recuperación de la dimensión afectiva

A mediados de los sesenta se publicó un trabajo de Devereux -un antropólogo y psicoanalista, además de conocedor profesional de las matemáticas y física contemporáneas (La Barre, 1991)-, que tuvo bastante impacto tanto en la antropología psicológica como en la etnopsiquiatría (Devereux, 1991). Para nosotros, la importancia de este autor reside en la cercana conexión de sus preocupaciones con las búsquedas hacia las que apuntan los trabajos que se encuentran en la vertiente sociodemográfica antes referida, las sugerentes posibilidades a las que se dirige para profundizar en el estudio del comportamiento poblacional, e incluso para una necesaria reflexión epistemológica sobre el quehacer demográfico. Pero voy a centrarme aquí en aquellos puntos cuya conexión con la aproximación microsocial me parecen más evidentes.

La reflexión sociodemográfica ha puesto en duda, con toda razón, que los sujetos puedan responder a las preguntas planteadas en una encuesta al margen de sus afectos y su ideología; hablan de las relaciones personalizadas y empáticas que se dan entre el investigador y los investigados en los estudios microsociales, y de las dificultades para reconstruir la realidad examinada de manera no prejuiciada. En Devereux encontramos un camino para continuar en esa línea de pensamiento hasta llegar a ciertas alternativas de solución, complejas pero enriquecedoras.

Para empezar, nos muestra que los aspectos afectivos influyen no sólo en el comportamiento y en las respuestas de los sujetos estudiados, sino en las del investigador mismo. No es común que el científico, preocupado por la objetividad, la neutralidad y la racionalidad, reconozca el hecho incontrovertible de su interés afectivo personal por su material; pero éste se manifiesta desde la elección misma del tema, los aspectos a los que dedica mayor atención, y algunas deformaciones que afectan su versión del fenómeno estudiado (Bastide, 1973). Por muy independiente que aparezca, en el nivel manifiesto, el objeto de estudio de la personalidad del científico, Devereux (1991) afirma que toda investigación es autopertinente en el nivel inconsciente; e incluso llega a representar -en forma más o menos indirecta- una introspección, en especial cuando se trata del estudio del comportamiento humano.

De hecho, la posibilidad de identificación del investigador con otros hombres -aquellos a quienes estudia-, aun si pertenecen a otras culturas, existe gracias a la unidad psíquica fundamental de la especie humana y a la capacidad del investigador 
para reconocer en cada sistema cultural, un caso particular del fenómeno humano genérico que es la cultura. Pero esta misma condición -la pertenencia del investigador y de los sujetos a la especie humana-determina también su vulnerabilidad frente al material que investiga. Lo asuma o no, el estudio de las personas observadas inevitablemente toca elementos que lo involucran, y eso es una fuente de ansiedad que puede impulsarlo a rehuir, de manera automática e inconsciente, determinados aspectos de la realidad que examina. ${ }^{5}$ La inhibición del afecto y la negación de las reacciones defensivas inconscientes, sin embargo, sólo logran una temporal supresión de la experiencia consciente de la ansiedad; puede incluso llegarse a una ilusión de mayor objetividad, pero lo que en realidad ocurre es una disminución de las posibilidades de dominar las propias propensiones y un incremento en la distancia sociopsicológica con los sujetos estudiados, con la consecuente distorsión en la percepción del problema analizado. La auténtica objetividad no se logra con la distancia afectiva -dice Devereux, siguiendo a Freud-, sino con el manejo consciente y creativo de las reacciones irracionales. En lugar de vaciar a la realidad de su contenido ansiógeno, lo que sugiere es convertir esa ansiedad ocasionada por el material de trabajo en un dato científicamente utilizable; la dimensión afectiva del observador puede convertirse, entonces, en piedra angular para la comprensión del comportamiento humano. 6

La condición para que eso sea posible es que el investigador pueda enfrentar y manejar adecuadamente sus propias reacciones ante el material observado, esto es, que logre comprender y manejar ese peculiar fenómeno denominado contratransferencia. 7 Dice La Barre que el antropólogo (y podríamos extender la afirmación a

5 Entre los mecanismos defensivos que ilustra Devereux, generados por el enfrentamiento con material ansiógeno, están la omisión de ciertas porciones de la información, su minimización, desaprovechamiento, malentendimiento, descripción confusa o ambigua, exageración, ciertas formas de reordenamiento, y también la utilización de la metodología -incluso la mejor- de manera defensiva, como "ataráxico", para poner distancia afectiva entre el investigador y un material que despierta ansiedad (Devereux, 1991; Bastide, 1973).

6 Este es un planteamiento cuyos orígenes se remontan, desde luego, a los trabajos originales de Freud, pero la discusión ha resurgido recientemente incluso entre quienes practican el psicoanálisis como procedimiento terapéutico (Casement, 1985; Stolorow y Atwood, 1992; Strean, 1992, entre otros).

7 Contratransferencia sería, dicho en términos simples, lo que sucede dentro del investigador ante los sujetos a los que observa; el conjunto de las distorsiones en la percepción del material que analiza; sus reacciones ante ese material, que lo llevan a responder y actuar de cierta forma en función de sus necesidades inconscientes, deseos y fantasías (Devereux, 1991). 
cualquier estudioso del comportamiento humano) es al mismo tiempo investigador, portador de cultura y persona, así que si desea que su trabajo vaya más allá de un relato sobre la manera en que él siente lo desconocido, su ciencia tendrá que disciplinarse por la conciencia de la contratransferencia (La Barre, 1991: 13-14). He ahí un primer punto en el que los estudios de población -en la medida en que se interesan por el estudio científico del comportamiento, $y$ muy en particular los que trabajan desde una perspectiva microsocial- pueden beneficiarse de los aportes de la teoría psicoanalítica.

La reflexión sociodemográfica ha cuestionado, como veíamos, que las personas entrevistadas compartan -o comprendan siquiera- la problemática que el investigador les impone a través de un cuestionario estructurado (Oliveira y García, 1986). Se ha preocupado también por descubrir la lógica de las acciones de los sujetos dentro de sus propias redes de significados, y por ajustar las interpretaciones de sus hallazgos al sentido que los sujetos le otorgan (Jelin et al., 1986).

Uno de los ejes básicos del planteamiento de Devereux podría ayudarnos a abundar en esa discusión: el reconocimiento de que el estudio del comportamiento humano se da en el contexto de una relación recíproca entre observador y observado, en donde el segundo está muy lejos de ser objeto pasivo, ignorante de su participación en el estudio, e indiferente a ella. Por el contrario, junto con el investigador participa en lo que el autor llama una díada observacional, en la cual cada uno es consciente de sí mismo, observa al otro, y sabe que es observado.

Veamos más de cerca las implicaciones de esta situación. El "investigador" y los "sujetos de estudio" no se distinguen porque uno observe y los otros sean observados, puesto que, como decíamos, todos se observan mutuamente y reaccionan ante lo que perciben. Se distinguen, entonces, sólo a partir de una definición operacional que es la que determina a quién se le permite hacer, pertinentemente, enunciados ("y esto percibo"), y a quién le corresponde hacer, pertinentemente, enunciados sobre enunciados ("Y esto percibo. Pero además, percibo que percibo y también percibo que el sujeto observado percibe"). Devereux identifica dos implicaciones metodológicas importantes de este reconocimiento de que los sujetos también observan al investigador: la primera es que este último sabe que él mismo, junto con sus técnicas de recolección de información, forma parte del "estímulo" ai que los entrevistados responden. Y la segunda, inmediatamente derivada de la anterior, es que el investigador intentará conocer cuál es su valor como "estímulo" para los sujetos estudiados -lo 
cual suele depender de su edad y género, su aspecto, su forma de ser, la situación ocupacional con la que se presenta e incluso el instrumental con el que trabaja-, para poder evaluar las reacciones que tienen ante él los sujetos y saber cuál es la parte de su mundo a la que le han dado acceso, por el significado que le han asignado.

La presencia del observador no tiene que tratarse, entonces, como una lamentable fuente de perturbación que habría que intentar (inútilmente) suprimir, sino que debe enfrentarse tal como realmente es, en la situación de investigación: una "perturbación" que ocasionará ciertas reacciones, y simultáneamente un "instrumento de observación" cuyas capacidades humanas son una fuente indispensable de datos complementarios y pertinentes para el estudio del comportamiento.

Como Devereux nos hace recordar, Freud demostró que aquellas situaciones de investigación en las que tanto el observador como el observado son humanos, no requieren de maniobras defensivas, sino de un manejo y aprovechamiento consciente y racional de ese hecho, por lo demás inevitable. Pero además constató que, dada la humanidad común del observador y el observado, el mejor y más adecuado instrumento u "órgano sensorio" del que podría disponerse es, justamente, su inconsciente, incluida la dimensión afectiva; de ahí que "... la manera más fructífera de estudiar al hombre [sea] por mediación de nuestra propia condición de humanos" (Devereaux, 1991: 199). Concluimos, también por esta vía, que la reintroducción del afecto en la investigación no es un obstáculo indeseable, sino un "instrumento" necesario cuyo adecuado manejo, lejos de hacernos perder objetividad, nos ayuda a ganarla.

Un tercer punto de contacto -y el último al que haré referencia- entre la investigación sociodemográfica y los aportes de Devereux, es el que se refiere a la interpretación de los mensajes que los sujetos expresan, su veracidad o exactitud, el universo de discurso al que pertenecen. Este punto abarca un amplio conjunto de posibilidades. Veamos algunas de ellas.

Las contradicciones entre las respuestas obtenidas mediante la aplicación de algunas encuestas demográficas y el comportamiento observable de las personas han sido objeto de muy diversas interpretaciones. Pero una de las posibles explicaciones es la que ilustra Devereux: que las afirmaciones se refieran a distintos universos de discurso (el de la opinión personal, o el de la opinión del grupo de pertenencia, o lo que se dice al investigador de acuerdo con el papel que se le ha asignado, o el diagnóstico especializado de una situación, o la reacción emocional ante algo, en- 
tre otros). De ser así, un más cuidadoso y consciente diseño de estas aproximaciones permitiría zanjar esa clase de confusiones y aclarar el sentido de las respuestas obtenidas.

Otra posibilidad es la que deriva de una de las cuestiones planteadas por ciertas corrientes sociodemográficas, que admiten que no sólo lo que los entrevistados informan requiere la atención del investigador, sino también lo que ocultan o declaran falsamente. Gracias al trabajo de Freud sabemos que lo que las personas dicen y las manifestaciones observables de su comportamiento pueden contener distintos tipos de mensajes: además de los manifiestos, expresados explícitamente, hay otros implícitos, latentes, que se comunican sin advertirlo, como si fueran huellas dejadas involuntariamente, cuya única evidencia es, en ocasiones, algo que no se dijo, una omisión o un olvido. Es ahí donde la teoría psicoanalítica interviene para permitir esa especie de lectura entre líneas que requiere la interpretación de tales mensajes. ${ }^{8}$

Pero hay una posibilidad más interesante aún, enteramente específica del psicoanálisis e íntimamente vinculada con los dos primeros puntos discutidos a lo largo de esta sección, sobre la importancia de que el investigador reconozca y pueda manejar adecuadamente las reacciones que le genera el material de investigación, y su capacidad para conocer el significado que los sujetos estudiados le confieren, así como sus propias reacciones ante ello. Se trata del aprovechamiento de las perturbaciones que ocasiona en el inconsciente del investigador lo dicho por el entrevistado. ${ }^{9}$

Veamos qué es lo que esto significa. La información que producen los sujetos llega al observador a través de sus canales sensoriales y se dirige a dos niveles de percepción: a la esfera consciente, en donde se maneja de manera racional, y a la esfera inconsciente, en donde puede despertar ciertas reacciones de ansiedad. En ese momento el observador puede sentirse inclinado a interrumpir el flujo de información procedente de las personas estudiadas, diciéndose quizá a sí mismo que ha averiguado ya todo lo que le interesa, y sin advertir que realmente se ha detenido en el punto en el cual dejó de encontrarse en condiciones de tolerar la ansiedad generada por el material. La teoría psicoanalí-

8 La situación psicoanalítica está ideada en forma tal que magnifica, y hace así más distinguibles, esos mensajes involuntarios. Nuestro estudio parte del supuesto de que el instrumento básico de recolección al que nos referiremos en la siguiente sección, el cuestionario interpretativo, puede constituir un estímulo desencadenante para ese tipo de mensajes, incluso sin pretender constituirse en una situación como la que se da en la terapéutica psicoanalítica.

9 Casement (1985) lo ilustra para la situación terapéutica. 
tica sugiere otro camino: considerar a esas perturbaciones que el observado produce dentro del investigador como información valiosa y aprovechable, adicional a la que se obtiene en el nivel racional y consciente. La idea es aproximarse al inconsciente de los sujetos estudiados -que no es directamente accesible al observador-a través del examen del inconsciente del investigador. ${ }^{10}$ Esto requiere, por supuesto, que el investigador se encuentre preparado para permitir que la información obtenida repercuta sobre su propio inconsciente, para examinar esa perturbación y para poder extraer de ella, mediante un cuidadoso análisis, datos relevantes. Esa capacidad del investigador para "escuchar" sin distorsión los mensajes que provienen de los sujetos que estudia, sin perder objetividad a causa de ciertas determinaciones intrapsíquicas que lo inducirían a deformar su material (en particular en aquellas áreas en las que ese material suscita ansiedad), depende en buena medida del adecuado manejo de la contratransferencia. De eso depende, también, la ya referida posibilidad de cambiar radicalmente el papel que se otorga a "lo subjetivo", para que en lugar de ser un elemento que ocasiona error sistemático se constituya en una importante fuente de información.

Cerraré esta reflexión sobre la vertiente psicoantropológica señalando que todas estas posibilidades se fundan, evidentemente, en algunos supuestos que pertenecen a la base de la teoría psicoanalítica. Ya antes hicimos referencia a uno de los principales, que varias corrientes antropológicas comparten con el psicoanálisis (Lutz y White, 1986): el de la unidad psíquica fundamental de la especie humana. Esta unidad no implica, de ninguna manera, uniformidad, sino por el contrario, una enorme flexibilidad, mayor aún que la que se observa en otras especies biológicas. Esta flexibilidad -que junto con la capacidad para crear cultura se considera como una de las características distintivas de la especie-, estaría dada por las muy diversas maneras en que cada cultura particular plasma sus huellas en la configuración de la psique humana, como lo ilustran los variables patrones de expresión emocional, de estímulos que desencadenan tales emociones, de situaciones sociales en las que es permitido expresarlas y de defensas culturalmente configuradas para inhibir su erupción (Lutz y White, 1986; Ekman, 1984; Shweder y LeVine, 1984; entre otros).

10 Devereux (1991) hace la analogía de que esta forma de acceder al inconsciente de los sujetos sería similar a lo que ocurre con la temperatura de ebullición del agua, que no es directamente observable sino que sólo se hace perceptible a través de un instrumento como el termómetro, o del examen de su aspecto al entrar en ebullición. 
Pero, en contraste con el nivel consciente, que suele ser altamente diferenciado y varía en alto grado entre un individuo y otro, el inconsciente constituiría una función de la psique relativamente indiferenciada, con grandes similitudes entre los diversos integrantes de la especie humana. Esa semejanza sería uno de los más importantes fundamentos para las posibilidades referidas.

La vertiente sociopsicoanalítica: explorando algunos de los vínculos entre el individuo y la sociedad

Uno de los puntos cuya solución ha preocupado de manera primordial a la investigación sociodemográfica, como veíamos, es el de los enlaces entre individuo y sociedad (Lerner y Quesnel, 1986). Esta pregunta establece la necesidad de incursionar en el problema de las relaciones entre el contexto sociocultural en el que se estructuran las relaciones interpersonales y la dimensión intrapsíquica de cada individuo como integrante del grupo social al que pertenece (familia, grupo étnico o religioso, localidad, región, nación). Esto nos lleva una vez más, aunque por otra vía, a la encrucijada entre la visión antropológica, la sociológica y la psicoanalítica. En este sentido hay algunos avances que iluminan varios de los aspectos involucrados en esa compleja relación (Obeyesekere, 1981; Ewing, 1991; entre otros). Pero quiero centrarme en una tradición más antigua, que pese a la polémica que suscitó (Jay, 1991) ha generado resultados interesantes y me parece un enlace muy sugerente entre esas tres disciplinas. Tal es la vertiente sociopsicoanalítica.

Esta teoría psicoanalítica, que forma parte del conjunto englobado en el llamado modelo relacional (Greenberg y Mitchell, 1983), toma como punto de partida los planteamientos freudianos sobre el carácter como instancia psíquica que determina la manera en que los sujetos tienden a actuar, de forma espontánea y relativamente permanente, ante diversas situaciones, y lo considera como una clave fundamental para explicar el comportamiento humano. Pero desarrolla una nueva orientación sociológica para la caracterología freudiana (biológicamente orientada), al proponer que no son las contingencias por las que pasa el desarrollo libidinal durante la maduración del ser humano lo que determina el carácter, sino que éste es una entidad psíquica que se configura a partir de las formas específicas de relación del individuo con el resto del mundo y consigo mismo (Fromm y Maccoby, 1990; Fromm, 1991).

Freud descubrió la capacidad del hombre para distorsionar la realidad de su propia experiencia con el objeto de conformarse a 
las normas socialmente establecidas, transmitidas por la familia, el grupo social y la cultura en la cual nace. El proceso de socialización conduce a que vastas áreas de la experiencia de los integrantes de cada sociedad queden desautorizadas, y tengan que racionalizarse y encubrirse por medio de complejos procesos, entre ellos el de la represión. Pero el límite del planteamiento freudiano fue que intentó extrapolar una teoría universal y unidimensional de las motivaciones, elaborada mediante la observación de la cultura victoriana de su época, y eso llevó a suponer que el centro del problema estaba en ciertas fuerzas que surgían del individuo mismo (los impulsos instintivos) y no en las relaciones entre el individuo y el mundo social formado por otras personas (Greenberg y Mitchell, 1983: 105-107).

En esta reorientación sociológica de la caracterología freudiana, el carácter se entendió como el sustituto humano de los instintos animales ausentes, esto es, como una segunda naturaleza del hombre. Y más allá del carácter, como estructura altamente individualizada y única para cada individuo, se postuló que hay un conjunto de rasgos caracterológicos relativamente típicos para los integrantes de los grupos sociales, compartidos por la mayor parte de ellos porque han sido moldeados en esa especie de matriz que son las condiciones sociales, económicas y culturales que les son comunes, a lo que se denominó el carácter social (Funk, 1982; Fromm, 1991).

El carácter social es el núcleo de la estructura caracterológica común a la mayoría de los individuos de una cultura dada, ${ }^{11}$ y resulta de la manera en que el carácter individual que diferencía a unas personas de otras dentro de la misma cultura,12 es moldeado por los patrones sociales y culturales. La energía psíquica (considerada como un elemento genérico de la especie humana) se transforma en energía psicosocial (como algo específico de los hombres que viven en cada sociedad histórica concreta) a través del carácter social, lo cual se da por diversas mediaciones entre las cuales la familia juega un papel crucial, en tanto que constituye uno de los puentes o eslabones entre el individuo, su estructura psíquica y las redes culturales en las cuales transcurre su exis-

11 Idea que actualmente puede afinarse mucho más, a la luz de algunos trabajos antropológicos recientes que permiten caracterizar a los diversos subgrupos culturales en una retícula mucho más fina (Lomnitz, 1992), y abren con ello la posibilidad de avanzar hacia la comprensión de los matices que en cada uno de estos subgrupos adopta el carácter social.

12 Además depende de las características de la personalidad de los padres, del ambiente social específico en que cada sujeto se desarrolla, y de las diferencias constitucionales de cada individuo. 
tencia (Fromm, 1986 y 1991). Desde muy temprana época, ${ }^{13}$ esta corriente sugirió que:

La familia es el medio esencial gracias al cual la situación económica ejerce su influencia formadora sobre la psique del individuo. La tarea de la psicología social consiste en explicar las actitudes psíquicas y las ideologías compartidas, socialmente pertinentes $-\mathrm{y}$ en especial sus raíces inconscientes- en términos de la infuencia de las condiciones económicas sobre las ansias de la libido14 (Fromm, 1990: 182).

Así, las sociedades están estructuradas en forma tal que los individuos van adquiriendo una visión del mundo y de su lugar en este que les permite desear hacer lo que en esa sociedad particular en ese momento histórico se requiere; el resultado de este proceso es que, término medio, los ciudadanos desean comportarse como se espera que lo hagan. Estos tipos de carácter socialmente necesarios no se imponen a los individuos por fuerzas externas, sino que la familia moldea a sus nuevos integrantes de acuerdo con esta necesidad social (Greenberg y Mitchell, 1983: 109).

Pero el papel que juega la sociedad en la formación del carácter no se limita a la etapa infantil por mediación de la familia, sino que continúa a lo largo de la vida entera de sus integrantes, a través de una especie de "recompensas sociales" que privilegian el desarrollo de ciertos rasgos caracterológicos necesarios para la adecuada ("normal" o "sana") adaptación a las normas y prácticas que prevalecen en el entorno sociocultural. El contenido de la vida interna de cada individuo se encuentra, pues, íntimamente vinculado con el contexto histórico y cultural en el que vive, y los procesos sociales y culturales, a su vez, se encuentran relacionados con la búsqueda de soluciones a la condición humana en cada uno de estos contextos. Las estructuras caracterológicas moldeadas en las matrices socioculturales, sin embargo, tienen su propia inercia, por lo cual no es de esperar que los cambios en las condiciones

13 Como señalan Greenberg y Mitchell (1983: 106), Fromm se caracterizó por plantear con décadas de anticipación diversas cuestiones que serían popularizadas más tarde por otros autores, aunque diversas circunstancias condujeron a que su pensamiento fuera poco reconocido y en no pocas ocasiones, mal comprendido o mal interpretado.

14 El término "libido", procedente del texto original "Método y función de una psicología social analítica" que data de 1932, se conservó en la versión que aparece en la compilación de 1970 (la cita está tomada de la cuarta reimpresión de 1990). Pero en la revisión del texto para la publicación de 1970 el autor advierte que lo que en sus primeros trabajos denominaba "estructura libidinal" de la sociedad corresponde a lo que en sus elaboraciones teóricas posteriores identificó como carácter social (Fromm, 1990: 198). 
socioeconómicas y culturales sean seguidos de manera automática por cambios similares en la estructura de carácter de los integrantes de las distintas clases sociales en una sociedad determinada (Fromm, 1990: 221).16

El más importante instrumento de trabajo utilizado por los investigadores que han continuado desarrollando la tradición sociopsicoanalítica, cuyo origen se remonta a los años treinta con los pensadores de la escuela de Frankfurt (Jay, 1991), es el llamado cuestionario interpretativo. Una de sus más conocidas aplicaciones fue la que realizaron Fromm y Maccoby (1990) en los años sesenta en una aldea mexicana, pero existen otras experiencias más recientes, entre ellas las del propio Maccoby (1988) en Estados Unidos, la de Funk en Alemania (Ubilla, 1993) y la de Gojman (1993) y Millán (1993), en México.

El cuestionario interpretativo se aplica a los individuos y va dirigido a lo psíquico personal, pero su propósito es estudiar el carácter social. Las respuestas que origina remiten al entorno socioeconómico y cultural en el que viven los individuos que responden, como matriz en la que sus características personales se moldean. En dichas respuestas se expresan, en consecuencia, muchos de los elementos compartidos por el grupo al que pertenecen los entrevistados, además de ciertos rasgos del carácter individual.

La interpretación del cuestionario se realiza a partir del análisis de la totalidad de las respuestas recogidas para cada sujeto,16 porque los tipos caracterológicos que intenta descubrir se revelan en el conjunto completo de las respuestas de cada persona, que se compara además con el resto de los sujetos entrevistados (Jay, 1991). Uno de los aspectos más difíciles de la interpretación es, por cierto, la distinción entre la expresión de opiniones convencionales, normas y rasgos de carácter; esa es una de las razones por las cuales se requiere, como un marco imprescindible para la interpretación del cuestionario, un conocimiento amplio y preciso de las características históricas, socioeconómicas y culturales del grupo estudiado. Esto es lo que orienta para distinguir las respuestas en las que se expresan rasgos caracterológicos de aquellas que reflejan la normatividad vigente en el grupo o las opiniones de tipo convencional. Es posible observar también, gracias a la interpretación del cuestionario, ciertas discrepancias entre actitudes conscientes e inconscientes (Fromm y Maccoby, 1990).

15 Planteamientos de autores como Bourdieu $(1980,1983,1988)$ abren nuevas posibilidades de desarrollo a muchas de estas vías sugeridas por Fromm.

16 A diferencia de otros cuestionarios, aquí no se trabaja con las respuestas a cada pregunta por separado, desvinculada del sujeto que las dio. 
El cuestionario interpretativo ofrece una alternativa para superar un problema al que ya nos referimos antes. Cuando se comprende que las apreciaciones humanas pasan por la forma cultural de configurar las respuestas por distorsiones inconscientes, por recuerdos encubridores, por olvidos que pueden verse como manifestación de la represión de ciertas partes del material (Devereux, 1991), la pretensión de tratar la información de las personas entrevistadas como verdades más o menos fieles sobre sí mismos o sobre la situación que describen, resulta bastante ingenua. Este instrumento no pretende registrar opiniones "verídicas" o versiones enteramente precisas de los hechos. Lo que intenta es captar los principales rasgos de carácter en los que se expresan las regularidades de la forma en que cada persona se comporta en los diversos ámbitos de su vida, para orientar hipótesis sobre las motivaciones que subyacen a su manera de pensar, sentir y actuar. Se trabaja con un texto constituido por lo que el sujeto reconoce y presenta al entrevistador, con lo que declara en respuesta a esa "provocación" que es el cuestionario, con las congruencias e incongruencias en sus respuestas originadas por las mezclas, tensiones y equilibrios de sus rasgos de carácter dominantes y secundarios. Este texto, leído en su totalidad y dentro del conjunto ąue forma con el resto de los textos obtenidos de otros sujetos pertenecientes al grupo, revela una serie de elementos que permanecen relativamente ocultos (cuanto más inconscientes, más ocultos) para las personas entrevistadas. Cada individuo expresa determinadas características de sí mismo y del grupo al que pertenece (familia, comunidad, grupo sociocultural), de modo que al conocer a un número mayor de sus integrantes se van evidenciando las regularidades (tipos caracterológicos predominantes), sus variaciones, e incluso ciertas particularidades individuales. $17 \mathrm{El}$ conjunto de las respuestas a estas preguntas directas y francas es analizado, pues, en su sentido caracterológico más que en el nominal (Fromm, 1991).

\section{Conclusión}

Se ha dicho que la investigación sociodemográfica latinoamericana ha pasado por ciertas etapas durante la segunda mitad del si-

\footnotetext{
17 Pese a que, a diferencia de la clínica, que se ocupa esencialmente de esto último, este instrumento no pretende ir demasiado lejos en esa dirección, sino solamente averiguar los rasgos que cada individuo comparte con el resto y algunos que distinguen a ciertos sujetos.
} 
glo: durante los sesenta se identifica un énfasis en las explicaciones psicosociales, los atributos y las motivaciones individuales, con datos recabados a través de encuestas, y en los setenta, un predominio de la preocupación por las explicaciones sociales e históricas, con lo cual la encuesta pasa a ser una más entre las diversas fuentes de información utilizadas (Oliveira y García, 1986).

La propuesta de este estudio vuelve en busca de explicaciones psicosociales, pero bajo una perspectiva diferente, al menos en dos sentidos: en primer lugar, porque la exploración parte de un reconocimiento más profundo de la complejidad de la interrelación entre el investigador y los sujetos, y del significado de los diversos tipos de mensajes que estos pueden emitir. $Y$ en segundo, porque se plantea como una entrada complementaria -pero imprescindible- al análisis de las especificidades históricas del contexto socioeconómico y cultural en el que se gesta el comportamiento ooblacional, sin el cual carecería por entero de marco interpretativo. Al señalar que considero a esta una entrada complementaria quiero decir que el abordaje psicosocial fuera de un contexto histórico, socioeconómico y cultural específico, no tendría sentido. $Y$ al subrayar que la considero imprescindible para la comprensión integral del comportamiento poblacional, coincido con uno de los argumentos de Jelin et al. (1986): el valor de la aproximación microsocial para el necesario cuestionamiento y reformulación de las hipergeneralizaciones que suelen conducir a muchas deformaciones y, por esa vía, al estancamiento del conocimiento.

\section{Bibliografía}

Bastide, R. (1973), "Prefacio", en G. Devereux, Ensayos de etnopsiquiatría general, Barcelona, Barral.

Bourdieu, P. (1980), Le sens pratique, París, Ed. de Minuit.

- (1983), Campo del poder y campo intelectual, Buenos Aires, Folios. (1988), La distinción, Madrid, Taurus.

Casement, P. (1985), Aprender del paciente, Buenos Aires, Amorrortu.

Devereux, G. (1991), De la ansiedad al método en las ciencias del comportamiento, sexta edición, México, Siglo XXI Editores.

Ekman, P. (1984), "Expression and the Nature of Emotion", en K. Shcerer y P. Ekman (ed.), Approaches to Emotion, Hillsdale, Nueva Jersey, Erlbaum, pp. 319-343.

Ewing, K. (1991), "Can Psychoanalytic Theories Explain the Pakistani Woman. Intrapsychic Autonomy and Interpersonal Engagement in the Extended Family", en Ethos 19:2, Estados Unidos, American An- 
thropological Association, pp. 131-160.

Fromm, E. (1986), Etica y psicoanálisis, México, Fondo de Cultura Económica (Breviario 74).

(1990), La crisis del psicoanálisis, México, Paidos.

(1991), Anatomía de la destructividad humana, México, Siglo

XXI Editores.

- y M. Maccoby (1990), Sociopsicoanálisis del campesino mexicano, México, Fondo de Cultura Económica.

Funk, R. (1982), Erich Fromm: The Courage to be Human, Estados Unidos, The Continuum Publishing Company.

Geertz, C. (1992), La interpretación de las culturas, Barcelona, Gedisa.

Gojman, S. (1993), "Un vistazo al proyecto mexicano de investigación participativa sociopsicoanalítica en una comunidad minera", en El carácter social, su estudio, un intercambio de experiencias. Cuadernos IV, México, Seminario de Sociopsicoanálisis, Instituto Mexicano de Psicoanálisis, A.C.

Greenberg, J. y S. Mitchell (1983), Object Relations in Psychoanalytic Theory, Estados Unidos, Harvard University Press.

Jay, M. (1991), La imaginación dialéctica. Una historia de la Escuela de Frankfurt, México, Taurus.

Jelin, E., J. Llovet y S. Ramos (1986), "Un estilo de trabajo: la investigación microsocial", en Problemas metodológicos en la investigación sociodemográfica, México, PISPAL/EI Colegio de México, pp. 109-126.

La Barre, W. (1991), "Prefacio", en G. Devereux, De la ansiedad al método en las ciencias del comportamiento, México, Siglo XXI Editores, pp. 11-14.

Lerner, S. y A. Quesnel (1986), "Problemas de interpretación de la dinámica demográfica y de su integración a los procesos sociales", en Problemas metodológicos en la investigación sociodemográfica, México, PISPAL/EI Colegio de México, pp. 127-147.

Lomnitz, C. (1992), Exits From the Labyrinth, Estados Unidos, University of California Press.

Lutz, C. y G. White (1986), “The Anthropology of Emotions”, en Annual Review of Anthropology, mim. 15, pp. 405-436.

Maccoby, M. (1988), Why work?, Washington.

Martínez S., Carolina (1993), "Una caracterización sociodemográfica de Xochimilco en 1990", ponencia al Primer seminario internacional de investigadores de Xochimilco, UAM-X/INAH/UDUAL/CIESAS/PPEX/Delegación Xochimilco, México, junio de 1993.

Millán, S. (1993), "Metodología para la evaluación del cuestionario interpretativo que se emplea durante las sesiones en el seminario de sociopsicoanálisis", en El carácter social, su estudio,un intercambio de experiencias. Cuadernos IV, Seminario de Sociopsicoanálisis, Instituto Mexicano de Psicoanálisis, A.C.

Obeyesekere, G. (1981), Medusa's hair. An Essay on Personal Symbols and Religious Experience, Chicago, The University of Chicago Press.

Oliveira, O. y B. Garcia (1986), "Encuestas ¿hasta dónde?", en Problemas metodológicos en la investigación sociodemográfica, México, PISPAL/EI 
Colegio de México, pp. 65-80.

PISPAL (Programa de Investigaciones Sociales sobre Población en América Latina) (1986), Problemas metodológicos en la investigación sociodemográfica, México, PISPAL/El Colegio de México.

Przeworski, A. (1982), "Teoría sociológica y el estudio de la población: reflexiones sobre el trabajo de la comisión de población y desarrollo de Clacso", en Reflexiones teórico-metodológicas sobre las investigaciones en población, México, El Colegio de México.

Shweder, R. y R. LeVine, (1984), Culture theory: essays on mind, self and emotion, Cambridge University Press.

Stern, C. (1986), "Introducción", en Problemas metodológicos en la investigación sociodemográfica, México, PISPAL/El Colegio de México, pp. 7-9.

Stolorow, R. y G. Atwood (1992), Contexts of Being. The Intersubjective Foundations of Psychological Life. Londres, The Analytic Press.

Strean, H. (1992), Resolving Counter-resistances in Psychotherapy, Nueva York, Brunner/Mazel Pub.

Ubilla, E. (1993), "Presentación de la forma de evaluación de las entrevistas del proyecto alemán con ejemplos de la fase de análisis", en $E l$ carácter social, su estudio, un intercambio de experiencias. Cuadernos IV, Seminario de Sociopsicoanálisis, Instituto Mexicano de Psicoanálisis, A.C. 